\title{
Making the link between dorsal stream sensitivity and reading
}

\author{
Alison Kevan and Kristen Pammer \\ School of Psychology, The Australian National University, Canberra, Australia \\ Correspondence to Alison Kevan, The School of Psychology, The Australian National University, Canberra, Australia \\ Tel: +6I 26125 4582; fax: +6I 26125 0499; e-mail: Alison.Kevan@anu.edu.au
}

Received I7 December 2007; accepted I9 December 2007

\begin{abstract}
Different levels of dorsal stream functioning were teased apart to determine whether the observed deficits in dyslexic readers may exist as early as the retinal level, and to explore the relative contribution that the different aspects of dorsal processing may make to reading. The paradigm combining frequency doubled stimuli with endogenous cueing demonstrated that dyslexic readers possess a retinal level magnocellular deficit. Regression analyses provided
\end{abstract}

Keywords: dorsal stream, dyslexia, magnocellular, orthographic, reading evidence to suggest that different levels of dorsal processing relates to various aspects of reading skills, with low-level magnocellular $M(y)$ processing relating to reading accuracy and irregular word reading, and dorsal stream functioning relating to all aspects of reading skills, including nonword reading. NeuroReport 19: 467-470 (C) 2008 Wolters Kluwer Health | Lippincott Williams \& Wilkins.

\section{Introduction}

In addition to language-based impairments, many dyslexic readers also possess visual deficits specific to the magnocellular (M) or dorsal pathway. At present, the exact origin or aetiology of the deficit is unknown, making the link between a dorsal stream deficit and reading difficulty unclear. Therefore understanding the conceptual link between a dorsal stream deficit and reading requires an understanding of how functioning in the dorsal stream and the $M$ cells may underlie reading skills.

Originating in the retina, the $M$ pathway projects through the lateral geniculate nucleus and primary visual cortex (V1) where it intermixes with parvocellular input before feeding into middle temporal area (V5/MT) and onto the posterior parietal cortex [1], a region specialised for controlling attentional orienting to visual locations [2]. Despite the considerable crosstalk between neural pathways, the dorsal stream is primarily innervated by $M$ input [3].

Recent research is becoming more selective in understanding the relationship between reading and the dorsal pathway [4,5], and there is even debate regarding the existence of a dorsal stream deficit in dyslexia [6]. Given the functional breadth of the dorsal pathway [7,8], it is not unrealistic to suggest that different aspects of reading, such as speed, accuracy, phonology and orthography, may be mediated by different functional components of the dorsal pathway, and this may in fact be reflected in the diverse findings. For example, findings demonstrating that dorsal stream processing predicts phonological awareness [9-13], reading accuracy [14-16], orthographic coding [15-17] or, indeed, no relationship with reading may reflect the fact that the different tasks tapping dorsal functioning may be influential in different components of the reading skill. This study will allow us to start to understand in a more specific way how the dorsal pathway is related to reading, and subsequent reading failure.

Higher-order dorsal deficits have been demonstrated in dyslexia, with dyslexic readers exhibiting difficulties in performing tasks that require sequential shifts of attention to locate a target $[18,19]$. Consistent with this, tasks involving shifting of attention correlate with phonological awareness [9], suggesting that the higher-order processing carried out by the dorsal pathway may be a reliable predictor of phonemic awareness and, in turn, reading ability. Specific $M$ stream deficits in dyslexia are also reported at the retinal level $[9,18,20]$, with dyslexic readers being less sensitive than control readers in detecting the frequency doubling (FD) illusion - believed to be a retinotopic measure of magnocellular $[\mathrm{M}(\mathrm{y})]$ cell function [21] (although see Ref. [22]). The FD studies [9,18,20] were carried out using the Humphrey Instruments frequency doubling technology (FDT) Visual Field Instrument machine (Zeiss Humphrey Systems, California, USA), which presents FD stimuli to 17 different retinal locations across the screen. Therefore, in addition to assessing FD sensitivity, the FDT machine also requires the participants to covertly shift their attention from a central fixation point to the location where the FD stimulus is presented. Thus, the FDT machine has the dual requirements of attentional shifting and FD detection. The behavioural differences reported between normal and dyslexic readers, however, are explained only in terms of reduced sensitivity to the FD illusion. Therefore, it is possible that lowered sensitivity to seeing the FD illusion may be due to the dyslexic readers being unable to shift their attention to the location in time to see the grating pattern, rather than impairment in seeing the FD stimuli itself. If the FDT machine is to be a viable technology for detecting and/or discriminating for dyslexia, then this possibility must be ruled out. 


\section{The present study}

The main aim of this study is to modify the FDT task to tease out the attentional demands to determine whether the FDT findings reflect impairments in retinal level FD sensitivity rather than an attentional deficit, and to explore how the different aspects of the task may relate to reading. As dyslexic readers are reported to have impairments in shifting attention quickly to a target [23], centrally presented endogenous cues will cue the location where the grating is to appear, which will allow the dyslexic readers to shift their attention in time to see the stimuli. Fixed grating stimuli will also be used to examine whether the endogenous cueing is beneficial to the dyslexic readers. In addition to this, different subskills of reading will be tested; speed, accuracy, orthographic (irregular words) and phonological (nonwords), to determine whether there is a differential relationship between the subskills of reading and the components of the dorsal pathway.

\section{Materials and methods Participants}

Primary school children ( 29 boys and 43 girls) aged between 8.5 and 12.4 years of age participated. All children were native English speakers, with normal or corrected-to-normal visual acuity, and normal nonverbal cognitive functioning as determined by Raven's progressive matrices. Reading achievement was measured using the Neale Analysis of Reading Ability (NARA). Dyslexic readers were classified as reading impaired based on a mean reading lag of two or more years on the accuracy and/or speed scores of the NARA, with cognitive abilities within the normal range, and no contributing social, cognitive or somatic disturbances. This resulted in 24 dyslexic and 48 control readers.

To assess component reading skills, the children read aloud 30 irregular words and 30 pronounceable nonwords from the Castles and Coltheart battery [24]. Irregular words (e.g. colonel) were used to assess orthographic processing in the form of direct access to the mental lexicon and nonwords (e.g. torlep) to measure grapheme to phoneme conversion. Data for all the psychometric tests are shown in Table 1.

\section{Stimuli and procedure}

Two separate visual stimuli conditions, a FD and a Fixed condition were presented monocularly in separate blocks. The FD stimuli were adapted from the Humphrey Instruments FDT Visual Field Instrument (Zeiss Humphrey

Table I Descriptive statistics on the psychometric tests (values are mean $\pm S D$ )

\begin{tabular}{lccc}
\hline Measure (unit) & Control $(n=48)$ & Dyslexic $(n=24)$ & $t$-test (d.f.=70) \\
\hline Age (years) & $126.60 \pm 10.99$ & $130.04 \pm 9.41$ & $\mathrm{NS}$ \\
Raven's IQ & $110.02 \pm 12.63$ & $100.042 \pm 117.75$ & $*$ \\
Accuracy (months) & $8.85 \pm 18.64$ & $-33.50 \pm 6.72$ & $* *$ \\
Speed (months) & $13.35 \pm 18.19$ & $-18.38 \pm 17.99$ & $* *$ \\
Irregular words & $20.50 \pm 3.39$ & $14.79 \pm 4.27$ & $* *$ \\
Nonwords & $26.69 \pm 3.54$ & $17.58 \pm 6.27$ & $* *$ \\
\hline
\end{tabular}

Accuracy and speed scores indicate deviation (in months) from expected reading level for their chronological age.

$* P<0.05 ; * * P<0.001$

NS, Not significant.
Systems, California, USA), consisting of $0.25-\mathrm{c} / \mathrm{deg}$ vertical sine wave gratings presented within a square aperture on a grey background. Gratings were modulated at $50-\mathrm{Hz}$ counter phase flicker, resulting in a percept of a grating that is twice the spatial frequency of the actual stimulus [18,25]. The Fixed stimuli consist of $0.5-\mathrm{c} / \mathrm{deg}$ static gratings that were engineered to look like the product of the illusion, and have the same behavioural response requirement, to allow for comparisons to be made between the FD and fixed conditions. All visual stimuli were presented on a BenQ 19 inch colour monitor (Neihu, Taipei, Taiwan), driven by an Acer NVIDIA graphics card (Hsien, Taipei, Taiwan), with a screen refresh rate of $100 \mathrm{~Hz}$.

Stimuli were presented to one of five regions of the visual field measuring $35^{\circ}$ visual angle, presented centrally or in one of the four corners of the screen. Centrally presented stimuli were displayed at fixation and therefore did not require any attentional shifts, whereas peripheral stimuli required the participant to shift their attention $14^{\circ}$ from fixation to the stimulus location. A central fixation square subtending $0.5^{\circ}$ remained visible throughout the entire testing session. Each stimulus ( $10^{\circ}$ diameter square grating) was displayed for $720 \mathrm{~ms}$, including a 160 -ms ramped onset and offset (Fig. 1). The contrast of the stimulus at each location was manipulated according to a modified binary search threshold strategy [26], with contrast threshold determined by calculating the mean of the last upper and lower stimulus presentations, when the test range was less than $6 \mathrm{~dB}$ after six reversals. The range of possible threshold level values was between $0 \mathrm{~dB}(\sim 100 \%)$ maximum contrast (lowest sensitivity) and $40 \mathrm{~dB}(\sim 0 \%)$ minimum contrast (highest sensitivity).

Endogenous cues were randomly presented in approximately $50 \%$ of trials (cued condition) in both the FD and Fixed conditions to indicate target location. For peripheral stimuli, the cue consisted of a small black arrow subtending $0.7^{\circ}$ and presented $0.3^{\circ}$ from fixation pointing to the location of the peripheral target. Whereas a small black cross subtending $0.7^{\circ}$ presented at fixation was used to alert the participant that the target would be presented in the centre of the screen. Each cue was presented for $500 \mathrm{~ms}$, followed by a presentation of the target. The maximum time allowed to respond was $1000 \mathrm{~ms}$. The children were instructed to respond by pressing the spacebar as quickly as possible

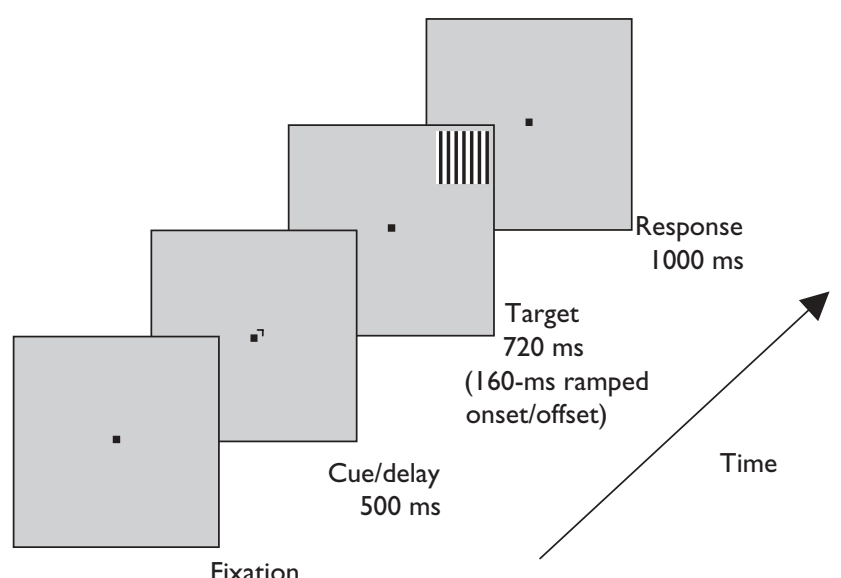

Fig. I Representation of the trial sequence (arrow represents the sequence and timing of events). 
whenever a target was detected at any of the five locations. Positive and negative catch trials were included, and no invalid cues were presented. To ensure the children were maintaining fixation and shifting attention covertly, eye movements were monitored by the Video Eyetracker (Cambridge Research Systems, England, UK). Any trial in which eye movements were larger than $10^{\circ}$ was discarded and replaced.

\section{Results}

\section{Reading groups}

Owing to IQ differences between reading groups $(P<0.05)$, analysis of covariance was conducted to factor out the effect of IQ; thus, all analyses reported here have IQ as a covariate. Collapsed over both eyes $(P>0.05)$, there were significant main effects for group $[\mathrm{F}(1,69)=8.85, P<0.005]$, stimulus $[\mathrm{F}(1,69)=22.04, P<0.001]$ and cue $[\mathrm{F}(1,70)=15.09, P<0.05]$; however, there was no significant main effect for position $(P=0.910)$. No significant interactions between group and position $(P=0.923)$, or between group and cue $(P=0.813)$ were found. Significant interactions were found between group and stimulus $[\mathrm{F}(1,69)=8.32, P<0.005)$; stimulus and position $[\mathrm{F}(1,70)=6.06, P<0.05]$; stimulus and cue $[\mathrm{F}(1,70)=7.78, P<0.05]$; and position and cue $[\mathrm{F}(1,70)=7.29$, $P<0.05]$; and a significant group by stimulus by cue threeway interaction $[\mathrm{F}(1,70)=7.04, P<0.01)]$.

Consistent with experimental predictions, planned comparisons revealed that dyslexic readers were significantly less sensitive to detecting FD stimuli, $t(70)=4.88, P<0.001$, whereas their thresholds for Fixed stimuli did not differ significantly from those of the control group $(P=0.192)$. The control group demonstrated no difference in cue benefit across the FD and Fixed conditions $(P=0.428)$; the dyslexic group, however, benefited more from the cue in the Fixed condition and demonstrated a larger cue effect when detecting the Fixed stimuli $(3.48 \mathrm{~dB})$ compared with the FD stimuli $(1.35 \mathrm{~dB}), t(23)=5.769, P<0.001$. Indeed, when the Fixed stimuli were cued, they showed greater sensitivity, suggesting that they were able to effectively use cue information. This was not the case in the FD condition, where cueing the FD stimuli did not increase their sensitivity (see Fig. 2).

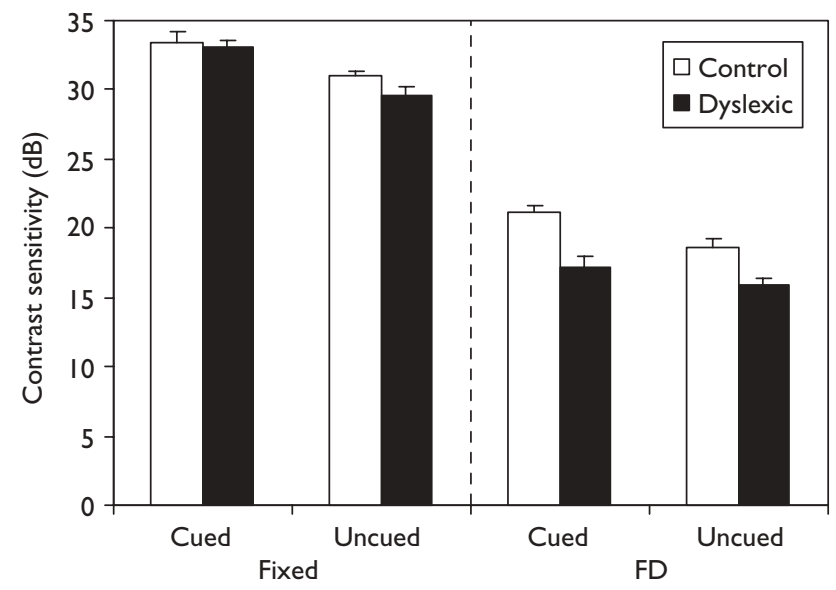

Fig. 2 Mean contrast thresholds $( \pm S E M)$ for the dyslexic and control readers in seeing the FD and Fixed stimuli [lower scores (dB) indicate poorer performance]. FD, frequency doubling.

\section{Regression analysis}

Hierarchical regression analysis was performed to assess the contribution that visual processing makes to reading subskills. As nonverbal IQ and Fixed sensitivity correlated significantly with FD sensitivity $(r=0.376$ and 0.407 , $P<0.001$ ), they were the first variables entered in each model. To dissect the relative contribution that FD detection and attentional shifting make to FDT sensitivity, cuedcentre-FD (low-level measure of FD sensitivity) and uncuedperipheral-FD (attentional shifting) were used as additional IVs. The models demonstrated that over nonverbal IQ and fixed sensitivity, cued-centre-FD makes a significant contribution to reading accuracy $\left[R^{2}\right.$ change $=0.570$, $\mathrm{F}(3,71)=10.922, P<0.001]$ and irregular word reading $\left[R^{2}\right.$ change $=0.219, \mathrm{~F}(3,71)=6.346, P=0.001]$. When uncued-peripheral-FD was added to the models, however, over and above low-level FD sensitivity, it was a significant predictor of all the reading subskills [accuracy: $R^{2}$ change $=0.374$, $\mathrm{F}(4,71)=10.020, P<0.001$; speed: $R^{2}$ change $=0.169, \mathrm{~F}(4,71)$ $=3.403, P=0.014$; irregular words: $R^{2}$ change $=0.278, \mathrm{~F}(4,71)$ $=6.444, P=0.001$; nonwords: $R^{2}$ change=0.280, $\mathrm{F}(4,71)=$ 6.527, $P<0.001]$. Thus, low-level FD processing (cuedcentre-FD) predicts reading accuracy and irregular word reading, whereas the attentional shifting component of the FD task (uncued-peripheral-FD) predicts all measures of reading subskills.

\section{Discussion}

Consistent with previous findings the dyslexic readers demonstrated a specific $\mathrm{M} /$ dorsal stream deficit, with dyslexic readers being less sensitive to seeing the FD illusion than the control readers, although being equally sensitive in detecting the Fixed gratings. This deficit is likely to stem from a low-level $\mathrm{M}(\mathrm{y})$ impairment and not because of the dyslexic readers being unable to quickly orient their attention to the stimulus location. Dyslexic readers demonstrated a cue effect in the Fixed conditions; however, the addition of endogenous cues to the FD task did not increase their sensitivity. The results therefore indicate that FD sensitivity represents a low-level retinal $\mathrm{M}(\mathrm{y})$ impairment in detecting the FD illusion.

Importantly, however, this study was also designed to provide some understanding as to how $\mathrm{M} /$ dorsal stream sensitivity might relate to reading or, by extension, reading failure. This was achieved by evaluating the relative contributions that higher-order attentional shifting and low-level FD detection make to different components of reading. Overall, the regression analysis indicated that $M$ / dorsal functioning was related to reading skills, however this is not a simple relationship. What has emerged is that different aspects of $\mathrm{M}$ /dorsal stream functioning (low-level $M$ vs. higher-order dorsal) seem to be differentially important to the various components of reading. Low-level $\mathrm{M}(\mathrm{y})$ functioning (sensitivity to the FD stimulus) accounted for a unique variance associated with reading accuracy and irregular word reading, suggesting that low-level visual analysis may be important for these specific aspects of reading. Attentional shifting, which is likely to reflect a higher-level, more integrated processing in the dorsal stream, additionally, however, predicts speed and nonword reading, thus reflecting a unique contribution to all measured aspects of reading. This is consistent with previous research linking dorsal functioning to 
both phonological [9-12,17] and orthographic processing $[5,13,14,16,17]$, and $M(y)$ cell functioning specifically to orthographic processing [15].

\section{Conclusion}

Results from this study support the notion of a M/dorsal stream impairment in dyslexic readers, and validate the use of the FDT instrument as a measure of low-level M(y) functioning. The results also highlight that the different levels of the $\mathrm{M}$ /dorsal pathway may contribute to different aspects of reading skills. Low-level $\mathrm{M}(\mathrm{y})$ sensitivity relates to reading accuracy and irregular word reading, whereas higher-order dorsal processing relates to reading speed and nonword reading in addition to reading accuracy, and irregular word reading.

\section{Acknowledgements}

The authors thank Andy Thomson for his programming expertise and Kristie Thorneywork for her help with testing. The contribution of the participating schools, including the staff, children and parents are also gratefully acknowledged. All our methods were performed in accordance with the guidelines set forth in the Declaration of Helsinki, and the experimental protocol was approved by the Human Research Ethics Committee on the Australian National University.

\section{References}

1. Merigan WH, Maunsell JHR. How parallel are the primate visual pathways? Annu Rev Neurosci 1993; 16:369-402.

2. Posner MI, Walker JA, Friedrich FJ, Rafal RD. How do the parietal lobes direct covert attention. Neuropsychologia 1987; 25:135-145.

3. Vidyasagar TR, Kulikowski JJ, Lipnicki DM, Dreher B. Convergence of parvocellular and magnocellular information channels in the primary visual cortex of the macaque. Eur J Neurosci 2002; 16:945-956.

4. Pammer K, Vidyasagar TR. Integration of the visual and auditory networks in dyslexia: a theoretical perspective. J Res Reading 2005; 28: $320-331$

5. Talcott JB, Gram A, van Ingelghem M, Witton C, Stein JF, Toennessen FE. Impaired sensitivity to dynamic stimuli in poor readers of a regular orthography. Brain Lang 2003; 87:259-266.

6. Skottun BC. On the conflicting support for the magnocellular-deficit theory of dyslexia. Trends Cogn Sci 2000; 4:211-212.

7. Vidyasagar TR. A neuronal model of attentional spotlight: parietal guiding the temporal. Brain Res Rev 1999; 30:66-76.
8. Born RT, Bradley DC. Structure and function of visual area MT. Annu Rev Neurosci 2005; 28:157-189.

9. Buchholz J, McKone E. Adults with dyslexia show deficits on spatial frequency doubling and visual attention tasks. Dyslexia 2004; 10:24-43.

10. Kinsey K, Rose M, Hansen PC, Richardson A, Stein JF. Magnocellular mediated visual-spatial attention and reading ability. NeuroReport 2004; 14:2215-2218.

11. Witton C, Talcott JB, Hansen PC, Richardson AJ, Griffiths TD, Rees A, et al. Sensitivity to dynamic auditory and visual stimuli predicts nonword reading ability in both dyslexia and normal readers. Curr Biol 1998; 8: 791-797.

12. Talcott JB, Hansen PC, Willis-Owen C, McKinnell IW, Richardson AJ, Stein JF. Visual magnocellular impairment in adult developmental dyslexics. Neuro-ophthalomology 1998; 60:187-201.

13. Cornelissen PL, Hansen PC, Gilchrist I, Cormack F, Essex J, Frankish C. Coherent motion detection and letter position encoding. Vis Res 1998; 38:2181-2191.

14. Cornelissen PL, Hansen PC, Hutton JL, Evangelinou V, Stein JF Magnocellular visual function and children's single word reading. Vis Res 1998; 38:471-482.

15. Pammer K, Kevan A. The contribution of visual sensitivity, phonological processing and non-verbal IQ to children's reading. Sci Stud Reading 2007; 11:33-53.

16. Talcott JB, Hansen PC, Assoku EL, Stein JF. Visual motion sensitivity in dyslexia: evidence for temporal and energy integration deficits. Neuropsychologia 2000; 38:935-943.

17. Talcott JB, Witton C, Hebb GS, Stoodley CJ, Westwood EA, France SJ, et al. On the relationship between dynamic visual and auditory processing and literacy skills; results from a large primary-school study. Dyslexia 2002; 8:204-225.

18. Pammer $\mathrm{K}$, Wheatley $\mathrm{C}$. Isolating the $\mathrm{M}(\mathrm{y})$-cell response in dyslexia using the spatial frequency doubling illusion. Vis Res 2001; 41:2139-2147.

19. Vidyasagar TR, Pammer K. Impaired visual search in dyslexia relates to the role of the magnocellular pathway in attention. NeuroReport 1999; 10:1283-1287.

20. Pammer K, Lavis R, Cornelissen PL. Visual encoding mechanisms and their relationship to text presentation preference. Dyslexia 2004; 10: 77-94.

21. Maddess T, Goldberg I, Dobinson J, Wine S, Welsh AH, James AC. Testing for glaucoma with the spatial frequency doubling illusion. Vis Res 1999; 39:4258-4273.

22. White AJR, Sun H, Swanson WH, Lee BB. An examination of physiological mechanisms underlying the frequency-doubling illusion. Invest Ophthalmol Vis Sci 2002; 43:3590-3599.

23. Brannan JR, Williams MC. Allocation of visual attention in good and poor readers. Percept Psychophys 1987; 41:23-28.

24. Castles A, Coltheart M. Varieties of developmental dyslexia. Cognition 1993; 47:149-180.

25. Kelly DH. Frequency doubling in visual responses. J Opt Soc Am 1966; 56:1628-1633.

26. Tyrrell RA, Owens DA. A rapid technique to assess the resting states of the eyes and other threshold phenomena: the modified binary search (MOBS). Behav Res Methods Instrum Comput 1988; 20:137-141. 